\title{
Regression of Late Onset Choroidal Metastasis from a Breast Carcinoma with Letrozole
}

\author{
Reinaldo Cancino ${ }^{a}$ José I. Vela ${ }^{a, c}$ Ivana Sullivan $^{b}$ \\ José A. Buil ${ }^{a}$ Carmen Alonso Muñoz ${ }^{b}$ \\ Departments of ${ }^{a}$ Ophthalmology and ${ }^{b}$ Oncology, Hospital de la Santa Creu i de \\ Sant Pau, and 'Institut Comtal d' Oftalmologia (ICO), Barcelona, Spain
}

\section{Key Words}

Intraocular tumor · Choroidal metastasis · Breast carcinoma · Letrozole

\begin{abstract}
We report the case of a 50-year-old woman with a history of diabetes mellitus who underwent left breast lumpectomy and ipsilateral lymphadenectomy in 1994 because of an infiltrating ductal carcinoma. Chemotherapy followed by radiotherapy to the breast and nodal areas were performed. In 2010, in a routine screening for diabetic retinopathy, two choroidal elevated masses above and below the optic nerve associated to serous retinal detachment of her right eye were noted. The patient was asymptomatic. Carcinoma was positive for hormone receptor. Hormone treatment with letrozole was established. Complete regression of the choroidal metastasis was observed 3 months later.

Ophthalmologic screening in asymptomatic patients with breast cancer has the advantage of being a noninvasive procedure and enables an early treatment in isolated cases. However, some studies are an argument against the usefulness of eye screening due to the low incidence of asymptomatic choroidal metastasis and the cost that involves performing it routinely in a large number of patients. Aromatase inhibitors are well-tolerated drugs that may be a powerful tool in the management of metastatic breast cancer that express hormone receptors.
\end{abstract}

\section{Introduction}

Uveal metastasis is the most frequent intraocular tumor and the choroid is the most commonly involved site [1]. Although breast carcinomas usually metastasize to the eye, the incidence of asymptomatic choroidal metastasis from disseminated breast cancer is low $[1,2]$. Eye screening in these patients remains controversial. 


\section{Methods}

A 50-year-old woman with a history of diabetes mellitus underwent left breast lumpectomy and ipsilateral lymphadenectomy in 1994 because of an infiltrating ductal carcinoma of $15 \mathrm{~mm}$ in diameter, which was classified as grade 2 . The estrogen and progesterone receptors were positive. The infiltrating ductal carcinoma was classified as a pT1N1M0, stage IIA. Cyclophosphamide, methotrexate and 5 -fluorouracil for 6 cycles followed by radiotherapy to the breast and nodal areas were performed. She continued treatment with tamoxifen for 5 years.

In 2000, the patient presented with an endometrial adenocarcinoma stage IIIA. Hysterectomy and bilateral oophorectomy were then performed. She received adjuvant chemotherapy with cyclophosphamide, doxorubicin and cisplatin scheme for a total of 3 cycles followed by pelvic radiotherapy and brachytherapy overdose.

In February 2010, in a routine screening for diabetic retinopathy, two choroidal elevated masses above and below the optic nerve associated to serous retinal detachment of her right eye were noted (fig. 1). The left eye was normal. The patient was asymptomatic, with a best corrected visual acuity of $20 / 20$ in both eyes. The ultrasound exam showed 2 masses located in the choroid with middle reflectivity and irregular internal structure without kappa angle or choroidal excavation. Fluorescein angiography showed a mottled hyperfluorescence in the areas of the lesions with fluorescein slight increase over time. Bone scintigraphy, thoracic computed tomography and abdominal echography were performed. A positive biopsy of the left axillary lymph node confirmed systemic recurrence after 16 years from diagnosis. Carcinoma was positive for hormone receptor but negative for Her2-Neu.

\section{Results}

Hormone treatment with letrozole and zoledronic acid was established. Complete regression of the choroidal (ig , 2) and lymph metastasis was observed 3 months later. At present, our patient shows a complete remission of her disease.

\section{Discussion}

The incidence of ocular metastasis from breast cancer ranges from 3 to $30 \%[3,4]$. It usually occurs 3-5 years after the diagnosis. Our patient developed recurrence 16 years from diagnosis, being asymptomatic at onset. In a review of 264 patients with choroidal metastasis from breast cancer, Shields and coworkers [1] found only a total of $7 \%$ of asymptomatic patients. In these patients attempts to demonstrate the usefulness of screening for choroidal metastasis have been done. Three studies conducted in patients with advanced disease have shown the potential role of the screening. The frequency of asymptomatic choroidal metastasis was 0, 0, and 5\%, respectively [4-6], increasing up to $11 \%$ in patients with more than one site of metastasis in the last study.

The frequency of choroidal metastasis has decreased in recent years. This can be explained by the improvement of the adjuvant therapy in the management of breast cancer (both chemotherapy and hormonal therapy) [7]. It is particularly effective in the eradication of metastasis of less than $1 \mathrm{~mm}$ in diameter in well-vascularized tissues such as the choroid.

Ophthalmologic screening in asymptomatic patients with breast cancer has the advantage of being a noninvasive procedure and enables an early treatment in isolated cases. However, the previously cited studies are an argument against the usefulness of 
eye screening due to the low incidence of asymptomatic choroidal metastasis and the cost that involves performing it routinely in a large number of patients.

Treatment of choroidal metastasis depends on several factors including the location, number and spread of primary tumor. Therapeutic options include systemic treatment (chemotherapy and hormone therapy), external beam radiotherapy (EBRT), local resection, photocoagulation, transpupillary thermotherapy, cryotherapy and enucleation.

Estrogen is an important hormone involved in the development and growth of breast tumors. Hormone therapy is used for those tumors that express hormone receptors. It has been found that $60-70 \%$ of breast cancers have estrogen receptors. In postmenopausal women, the main source of estrogen comes from peripheral conversion of adrenal androgen. The aromatase inhibitors are a group of drugs that act by preventing this conversion, decreasing circulating estrogen levels. New third generation aromatase inhibitors include anastrozole, letrozole and exemestane [8].

In a case series reported by Manquez et al. [9], 17 cases of uveal metastasis were treated with one of these hormonal therapeutic agents. Both systemic and choroidal metastasis regressed in 10 cases (59\% of cases) after a 20 -months follow-up.

In conclusion, aromatase inhibitors are well-tolerated drugs that may be a powerful tool in the management of metastatic breast cancer that express hormone receptors.

\section{Acknowledgement}

We thank C. Newey for revising the English language.

\section{Disclosure Statement}

The authors have no financial interest in any aspect of this report. 


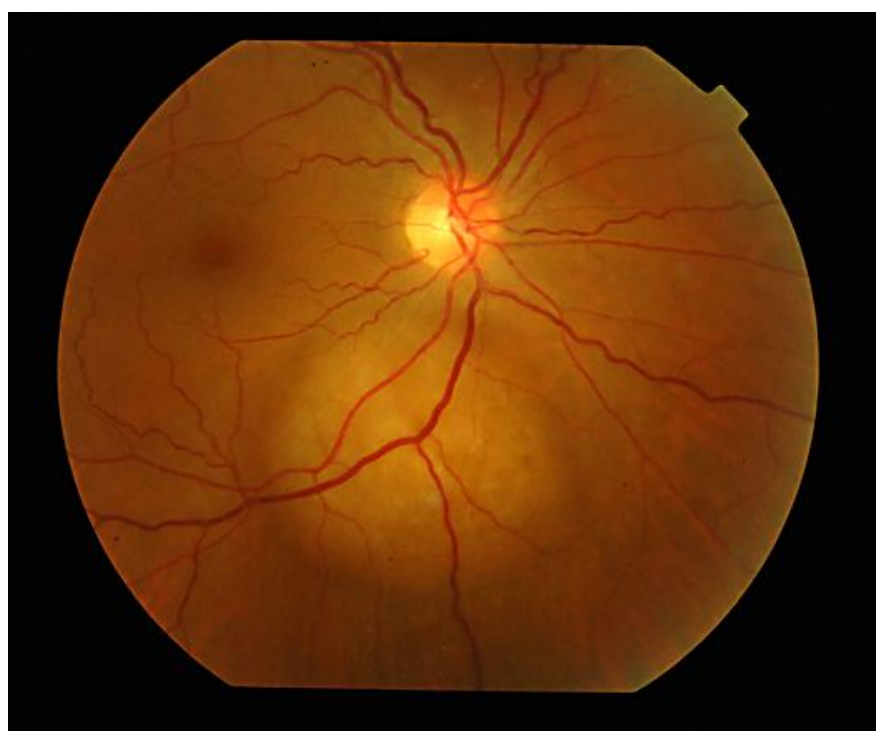

Fig. 1. Fundus photograph of the right eye showing a choroidal elevated mass below the optic nerve associated to serous retinal detachment.

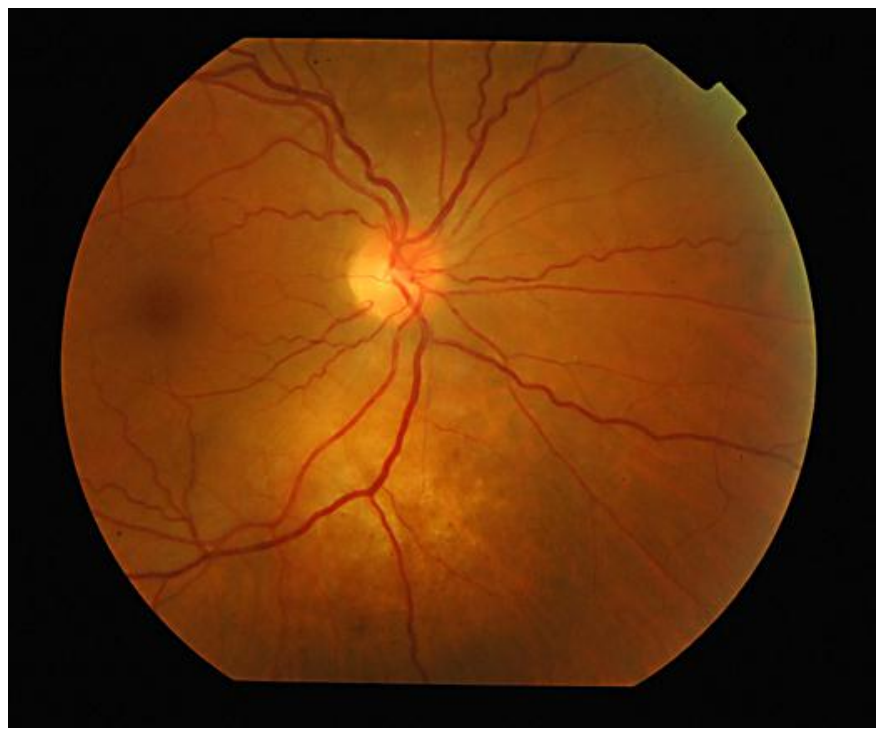

Fig. 2. Complete regression of the lesion was noted 3 months later.

\section{References}

1 Demirci H, Shields Cl, Chao AN, Shields JA: Uveal metastasis from breast cancer in 264 patients. Am J Ophthalmol 2003;136:264-271.

2 Mewis L, Young SE: Breast carcinoma metastasis to the choroid: analysis of 67 patients. Ophthalmology 1982;89:147-151.

3 Merril CF, Faufman DI, Dimitrov NV: Breast cancer metastatic to the eye is a common entity. Cancer 1991;68:623-627. 
-4 Fenton S, Kemp EG, Harnett AN: Screening for ophthalmic involvement in asymptomatic patients with metastatic breast carcinoma. Eye 2004;18:38-40.

-5 Barak A, Neudorfer M, Heilweil G, Merimsky 0, Lowenstein A: Decreased prevalence of asymptomatic choroidal metastasis in disseminated breast and lung cancer: argument against screening. Br J Ophthalmol 2007;91:74-75.

-6 Wiegel T, Martin K, Bornfeld N: Frequency of asymptomatic choroidal metastasis in patients with disseminated breast cancer: results of a prospective screening programme. Br J Ophthalmol 1998;82:1159-1161.

7 Jang RW, Doherty M, Hopkins JJ, Warner E: A case of prolonged disease-free survival in a patient with choroidal metastasis from breast cancer. Nat Clin Pract Oncol 2009;6:118-121.

$>8$ Smith IE, Dowset M: Aromatase inhibitors in breast cancer. N Engl J Med 2003;348:2431-2442.

-9 Manquez ME, Brown MM, Shields CL, Shields JA: Management of choroidal metastasis from breast carcinomas using aromatase inhibitors. Curr Opin Ophthalmol 2006;17.251-256. 